\title{
Laryngeal paralysis associated with a muscle pseudotumour in a young dog
}

\author{
Francesca Rizzo ${ }^{1, *}$, Cecilia Benetti ${ }^{1}$, Consuelo Ballatori ${ }^{1}$ and Diana Binanti ${ }^{2}$ \\ ${ }^{1}$ Clinica Veterinaria Colombo, Viale Colombo 153, 55041, Lido di Camaiore (LU), Italy \\ ${ }^{2}$ AbLab, Laboratorio di Analisi Veterinarie, Sarzana (SP), 19038, Italy
}

\begin{abstract}
An 18-month-old male entire Bloodhound dog was presented with a six-week history of progressive inspiratory dyspnoea, stridor, dysphonia and exercise intolerance. CT scan performed elsewhere had revealed the presence of an unencapsulated nodular mass $(3 \times 1 \times 5 \mathrm{~cm})$ dorsal to the larynx and first tracheal rings. Laryngoscopy demonstrated the presence of bilateral laryngeal paralysis and distorted laryngeal architecture suggestive of extraluminal compression. Histopathology results of incisional biopsies from the mass were suggestive of a benign non-neoplastic muscular lesion. Surgery was performed to manage laryngeal paralysis and attempt mass excision. A second histopathology examination confirmed an inflammatory and dysplastic lesion suggestive of a pseudotumour. All clinical signs resolved after surgery and at the 13 months follow-up the dog remains asymptomatic. To the authors' knowledge, this is the first report of a case of laryngeal paralysis caused by a muscle pseudotumour in a young dog.

Keywords: Dog, Laryngeal paralysis, Larynx, Muscle pseudotumour.
\end{abstract}

\section{Introduction}

Muscle pseudotumours are benign non-neoplastic lesions arising from the skeletal muscle (Cooper and Valentine, 2002; The Armed Forces Institute of Pathology, 2007). They have been reported in humans (Gude et al., 2011; Segawa et al., 2014) and occasionally in animals (Cooper and Valentine, 2002; van der Woerdt, 2008; Knight et al., 2009; Loderstedt et al., 2010). Aetiology of muscle pseudotumours remains unknown, although several hypotheses have been postulated. Laryngeal paralysis (LP) in dogs is the most common disease involving the larynx (MacPhail, 2014) and a frequent cause of upper respiratory obstruction and dyspnoea in large breed dogs. Acquired LP is most commonly reported in older dogs. It can be idiopathic or it may result from trauma, neuromuscular disease, iatrogenic injury or mass-related compression of the recurrent laryngeal nerve (Millard and Tobias, 2009). This paper describes clinical presentation, diagnosis, treatment and follow-up of a case of LP caused by a muscle pseudotumour in an 18-month-old Bloodhound dog.

\section{Case Details}

An 18-month-old, $47 \mathrm{~kg}$, male entire, Bloodhound was presented with a six-week history of progressive inspiratory dyspnoea, stridor, dysphonia and exercise intolerance. The dog had been submitted to medical therapy by previous vets with amoxicillin-clavulanic acid (Synulox; Pfizer), cephalexin (ICFVET; ICF), carprofen (Rimadyl; Pfizer) and prednisone (Deltacortene; Bruno Farmaceutici SPA) without significant clinical improvement. Previous CT scan investigation of the neck region performed by primary veterinary surgeon had revealed the presence of a space-occupying lesion $3 \times 1 \times 5 \mathrm{~cm}$ dorso-lateral to the trachea (Fig. 1).

On presentation the dog appeared bright and alert, and in good body condition. Tachypnoea, increased inspiratory effort and stridors were evident and more pronounced when the dog was excited. Remaining clinical examination was unremarkable, as was neurological examination and thorough palpation of the region of the neck, pharynx and larynx. Haematology, full serum biochemistry profile, thyroid profile and coagulation times were all within normal range. Thoracic radiographs and abdominal ultrasound were performed to rule out the presence of additional masses and did not reveal any significant findings. Direct and indirect laryngoscopy and tracheoscopy were performed under appropriate light anaesthesia. Propofol (Proposure; Merial Italia SpA) was slowly titrated intravenously in order to maintain spontaneous breathing and allow inspection of the larynx. These investigations revealed the presence of bilateral laryngeal paralysis; diffuse laryngeal oedema was also present, which extended to the proximal section of the trachea. The rima glottidis appeared of abnormal shape due to severe dorso-lateral compression suggestive of the presence of an extraluminal space-occupying mass at the right side (Fig. 2). The trachea was otherwise normal. Three incisional biopsies of the mass were obtained via a right lateral surgical approach to the neck region just dorsal to the larynx, facilitating direct visualisation of the area involved. 

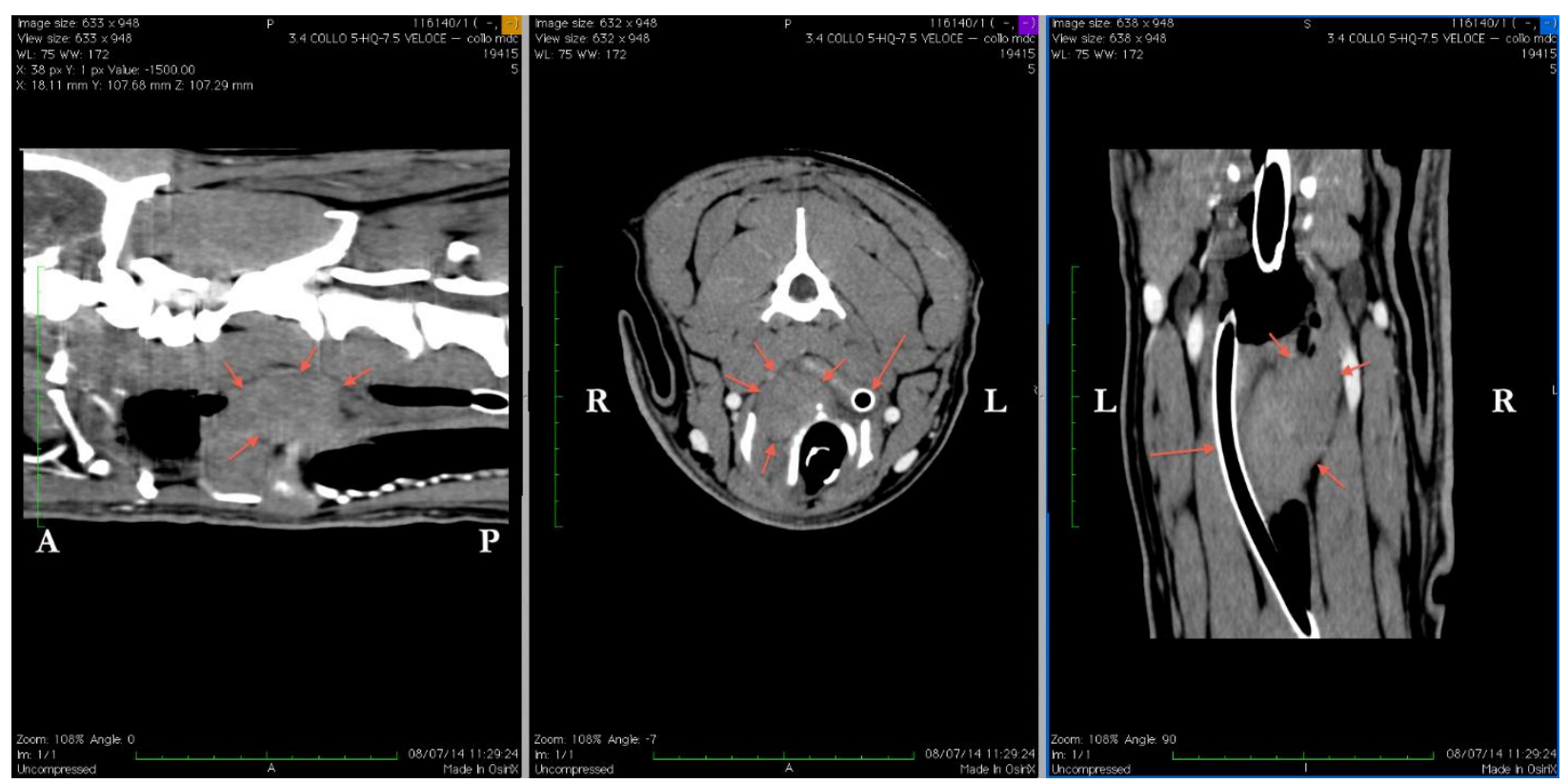

Fig. 1. CT scan images (from left to right: sagittal, transverse and dorsal plane): the mass-type lesion (short arrows) extends dorsally to the first tracheal rings and larynx, causing right lateral deviation of the proximal trachea and oesophagus; the deviation of the intraoesophageal tube appears evident (long arrows).

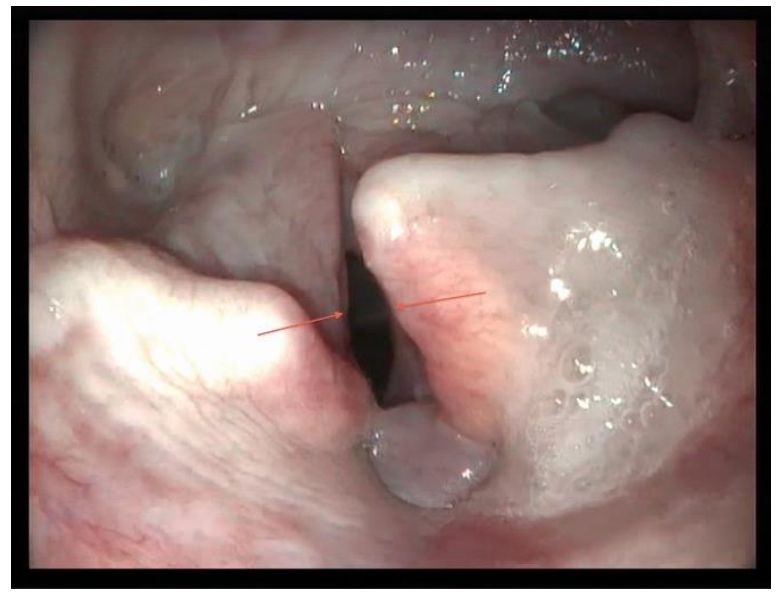

Fig. 2. Laryngoscopy image: The extraluminal mass distorts the normal laryngeal architecture causing narrowing of the glottis lumen (arrows) and bilateral laryngeal paralysis.

The abnormal tissue appeared to be muscle tissue slightly bulging, firmer and paler than the surrounding muscle on palpation. The dog recovered well from the procedure with the respiratory signs remaining unchanged.

Microscopically, the biopsy samples were represented by skeletal muscle tissue with severe degrees of dysplasia and moderate inflammation. The mass was composed of haphazardly arranged mature skeletal muscle fibers, with marked size variations, from hypertrophy to atrophy, and disarray of orientation, with some fibers in transverse section, longitudinal section, and others in oblique section.
Signs of regeneration, degeneration and necrosis of the fibres were present. Mild perimysial and endomysial fibrosis was evident. A mild multifocal inflammatory infiltrate was present and composed mainly by lymphocytes and plasma cells with scattered eosinophils and neutrophils. Findings suggestive of a neoplastic process were not evident.

Surgery was planned to manage laryngeal paralysis and perform mass removal. The patient was placed in right lateral recumbency and a unilateral cricoarytenoid lateralisation was performed (Kitshoff et al., 2013). The patient was then placed in left lateral recumbency for an optimal surgical approach to the mass. A skin incision was made dorsal to the jugular vein from the caudal margin of the mandible to the first tracheal rings. Blunt and sharp dissection through the subcutaneous muscles allowed the exposition of the dorso-lateral right laryngeal region. The mass was palpable and included the right caudo-lateral portion of the thyropharyngeal and cricopharyngeal muscles. The muscle portion involved was excised via skeletisation of the laryngeal cartilages. Haemostasis was achieved by bipolar cauterisation. Muscles, subcutaneous tissues and skin were closed routinely. Recovery from surgery was uneventful and clinical signs resolved immediately. Histopathological examination of samples obtained from the mass was similar to previous results of incisional biopsy examination (Fig. 3).

The patient was re-examined 12 days after surgery for suture removal and remains asymptomatic at follow-up 13 months after surgery. 




Fig. 3. Histology image: Portion of skeletal muscle with markedly distorted architecture and mild inflammatory changes. Myofibers show severe variations in size, shape and staining affinity. Disarray of orientation is evident, with fibres in transverse, longitudinal and oblique section. Degeneration, regenerative changes and fibrosis are also evident (H\&E stain, 10X).

\section{Discussion}

To the authors' knowledge, this is the first report of an acquired LP in a young dog caused by the presence of a muscle pseudotumour.

The term muscle pseudotumour includes a group of uncommon benign non-neoplastic masses arising from the skeletal muscle. Several synonyms exist in human literature (Knight et al., 2009), contributing to confusion surrounding the comprehension of this uncommon disease. This condition has been reported in humans and animals (horses, dogs and cats) with no particular age or sex incidence and with different localisations (Cooper and Valentine, 2002; van der Woerdt, 2008; Loderstedt et al., 2010; Gude et al., 2011; Segawa et al., 2014).

In humans, clinical or subclinical muscle injury and denervation injury have been proposed as a possible aetiology, however such correlations have not been proved (Cooper and Valentine, 2002; The Armed Forces Institute of Pathology, 2007; Knight et al., 2009). The lesion may grossly appear paler and firmer than the surrounding normal muscle tissue. Pain is not a usual feature of muscle pseudotumours. When possible, wide excision of the lesions can be curative but the mass may also recur (Cooper and Valentine, 2002; The Armed Forces Institute of Pathology, 2007). In this dog clinical signs caused by LP were the only signs associated with the presence of the mass. LP is more frequent in older dogs in its acquired form; congenital forms are uncommon and have been reported in certain breeds with usual onset of clinical signs at less than 1 year of age.

Causes of acquired LP can be several although in most dogs the cause remains undetermined and LP is classified as idiopathic (Millard and Tobias, 2009; MacPhail, 2014).

In this case report the patient was a young adult dog; the age was unusual for both a congenital or acquired form of LP. All the additional diagnostics were unremarkable except for the presence of a mass on the CT scan of the neck. The severe structural alteration of the larynx seen on laryngoscopy and caused by the presence of the mass justified the impairment of the physiological movement of the larynx. We could not exclude or confirm concomitant nerve damage; however the anatomical localisation of the mass was not compatible with compression of the recurrent laryngeal nerve. Unilateral cricoarytenoid lateralisation was performed to relieve respiratory signs because the clinical response to the surgical excision of the mass alone was unpredictable.

The lack of response to initial medical treatment could have been suggestive of a neoplastic mass rather than an inflammatory condition and may have mislead the decision making process. Although histology report from the initial biopsies was indicative of a nonneoplastic muscular lesion, the samples could have not been representative of the mass. In this case the type and extent of surgery was decided balancing preservation of function against completeness of excision. Surgical removal of the mass associated with surgical management of LP could be a viable and curative treatment for similar cases. In this case, at the 13 months follow-up, the dog remains well with no signs of recurrence. One limitation of this case is that re-evaluation on follow-up was only based on clinical and neurological examination, whilst a CT scan of the neck would have been a desirable option to assess any possible recurrence of the mass lesion.

Muscle pseudotumour associated with laryngeal paralysis in dogs has not been documented previously and, although a rare condition, it should be listed amongst the differential diagnosis of focal lesions and possible causes of LP in dogs.

\section{Aknowledgements}

The authors would like to acknowledge Dr Linda Mecattini from the Clinica Colombo for her assistance in performing endoscopy and Dr Simonetta Citi from the University of Pisa for her assistance in interpretation of the CT images. The authors confirm that all individuals personally acknowledged have given their permission to be listed.

\section{Conflict of interest}

The authors declare that there is no conflict of interests.

\section{References}

Cooper, B.J. and Valentine, B.A. 2002. Muscle Pseudotumors. In: Tumors in domestic animals, $4^{\text {th }}$ ed, ed Meuten D. J., Iowa state press, Ames, IA, pp: 359-361. 
Gude, D., Rayudu, R. and Bansal, D. 2011. How pseudo is an inflammatory pseudotumor? Indian J. Med. Paediatr. Oncol. 32, 204-206.

Kitshoff, A.M., Van Goethem, B., Stegen, L., Vandekerckhove, P. and de Rooster, H. 2013. Laryngeal paralysis in dogs: An update on recent knowledge. J. S. Afr. Vet. Assoc. 84, E1-E9.

Knight, C., Fan, E., Riis, R. and McDonough, S. 2009. Inflammatory myofibroblastic tumors in two dogs. Vet. Pathol. 46, 273-276.

Loderstedt, S., Walmsley, G.L., Summers, B.A., Cappello, R. and Volk, H.A. 2010. Neurological, imaging and pathological features of a meningeal inflammatory pseudotumour in a Maltese terrier. J. Small Anim. Pract. 51, 387-392.
MacPhail, C. 2014. Laryngeal disease in dogs and cats. Vet. Clin. North Am. Small Anim. Pract. 44, 19-31. Millard, R.P. and Tobias, K.M. 2009. Laryngeal paralysis in dogs. Compend. Contin. Educ. Vet. 31, 212-219.

Segawa, Y., Yasumatsu, R., Shiratsuchi, H., Tamae, A., Noda, T., Yamamoto, H. and Komune, S. 2014. Inflammatory pseudotumors in head and neck. Auris Nasus Larynx. 3, 321-324.

The Armed Forces Institute of Pathology. 2007. Department of Veterinary Pathology. http://askjpc.org/wsco/wsc/wsc07/07WSC01.pdf

van der Woerdt, A. 2008. Orbital inflammatory disease and pseudotumor in dogs and cats. Vet. Clin. North Am. Small Anim. Pract. 38, 389-401. 